\section{Thick Eccentric Circular Iris in Circular Waveguide}

S. P. Yeo and S. G. Teo

Abstract-Other researchers have observed that eccentric irises possess certain advantageous features that may lead designers to prefer them over the conventional concentric irises. The least-squares boundary residual method (LSBRM) is utilized in this paper to analyze the behavior of such an eccentric-iris structure (of nonzero thickness). Tests have confirmed that the computer model thus obtained is capable of yielding numerical results that are accurate to within $\pm 1 \%$.

Index Terms-Circular waveguides, modeling, waveguide discontinuities.

\section{INTRODUCTION}

A LTHOUGH circular irises have been in use since the earlier part of this century [1], [2], they still continue to attract the attention of researchers [3]-[6]. James, for instance, has noted that "there is a lack of readily available data on the admittance of circular irises in waveguides" [3, p. 430].

It is common practice to place circular irises concentrically within circular waveguides. However, Shen and MacPhie have recently pointed out that "the off-centered iris has more design flexibilities than the centered iris and provides the advantage of a larger susceptance range" [6, p. 2641]. The model developed by them to predict the characteristics of this thick eccentric-iris structure is based on the conservation of the complex power technique (CCPT), but unfortunately they have "assumed that no propagating modes exist in the smaller waveguide" [6, p. 2640] (which is the $-1 / 2 t<$ $z<1 / 2 t$ section in Fig. 1), and the validity of their model is thus limited to a narrow frequency range. We have chosen instead to develop a different model-based on the least-squares boundary residual method (LSBRM), which is noted for its mathematical rigor [7] - which does not require such an assumption; in fact, our model even allows the possibility of the $-1 / 2 t<z<1 / 2 t$ iris region supporting more than one propagating mode.

\section{Development of Model}

The symmetry of the overall structure about the $z=0$ midplane suggests that we can resort to an eigenmode approach. We are, therefore, able to reduce the actual two-port, depicted in Fig. 1, to the equivalent one-port, sketched in Fig. 2, where the input $p_{0}$ wave from the source excites the various $p_{1}, p_{2}, p_{3}, \cdots$, waves in the circular waveguide (Region I, where $z<-1 / 2 t$ ) and $q_{1}, q_{2}$, $q_{3}, \cdots$, waves within the bisected-iris aperture (Region II, where $-1 / 2 t<z<0$ ). If the iris is subjected to odd-eigenmode excitation, each of the $q_{1}, q_{2}, q_{3}, \cdots$, waves launched from the $z=-1 / 2 t$ discontinuity plane will be reflected from the effective short circuit at the $z=0$ midplane with a phase change of $\pi$. On the other hand, for the even-eigenmode case, there will be an open circuit instead over the $z=0$ aperture and the corresponding reflection coefficient for each of the $q_{n}$ waves becomes +1 . Equation (1), shown at the bottom of the following page, provides a suitable measure of the total field mismatch existing over the transverse plane at $z=-1 / 2 t$ (i.e.,

Manuscript received May 9, 1997; revised May 11, 1998.

The authors are with the Department of Electrical Engineering, $\mathrm{Na}$ tional University of Singapore, Kent Ridge, Singapore 119260 (e-mail: eleyeosp@nus.sg).

Publisher Item Identifier S 0018-9480(98)05495-7.

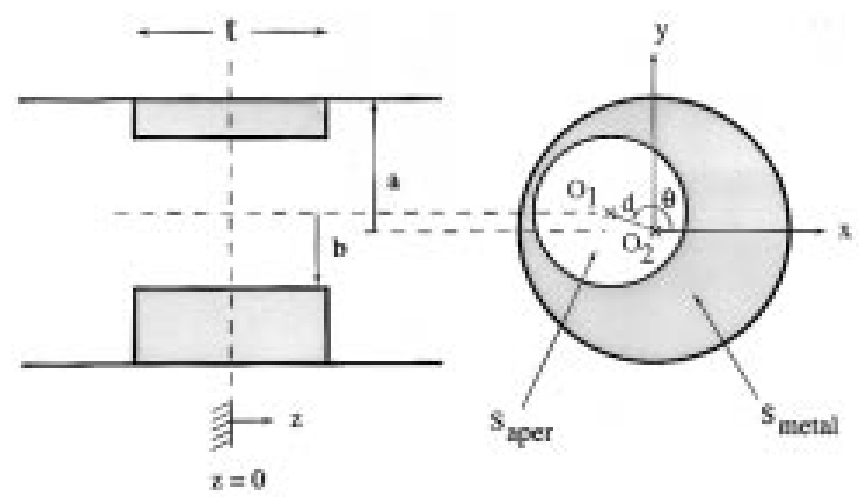

Fig. 1. Thick eccentric circular iris (with axis at $O_{1}$ ) in circular waveguide (with axis at $O_{2}$ ).

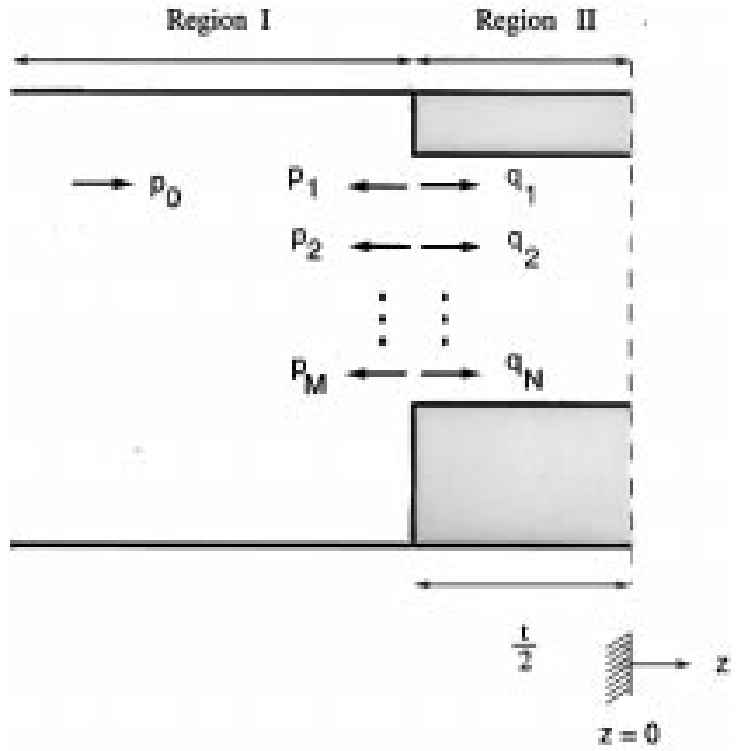

Fig. 2. Equivalent one-port structure for use in eigenmode analysis of iris depicted in Fig. 1.

over both aperture interface $S_{\text {aper }}$ and metallic surface $S_{\text {metal }}$ ), where $\gamma_{n}$ is the propagation constant of the mode associated with the $q_{n}$ wave in Region II, $\delta$ is the Kronecker delta, $Z$ is some appropriate impedance term (which we have found, from our computational trials, should be assigned the value of $1 \mathrm{k} \Omega$ ), $K$ is an integer variable that has an odd value for the odd eigenmode and even value for the even eigenmode, and $\underline{e}$ and $\underline{h}$ are the transverse components of the electric and magnetic fields, respectively, for each of the modes (with the superscripts "I" or "II" denoting either of the two regions). In principle, complete expansions should be used to represent the fields in Regions I and II; however, in practice, both $M$ and $N$ have to be assigned finite values in view of the constraints imposed by the computational facilities available.

As has been explained in [7]-[9], it is more convenient to recast the residual defined in (1) as a positive-definite Hermitian form

$$
\Delta=\sum_{i=1}^{i=M+N+1} \sum_{j=1}^{j=M+N+1} y_{i}^{*} w_{i j} y_{j}=\underline{y}^{*} \underline{W} \underline{y}
$$


where $y=\left(p_{0} p_{1} p_{2}, \cdots, p_{M} q_{1} q_{2}, \cdots, q_{N}\right)^{t}$ and $\underline{W}$ is a $(M+N+$ 1) $\times(\bar{M}+N+1)$ positive-definite Hermitian matrix containing the coupling integrals between the various modes of the expansions. We have been able to derive closed-form expressions for all the matrix entries of $\underline{W}$ (where, as in [6], Graf's addition theorem has been utilized to effect the transformation of the cylindrical-coordinate system from that based on $O_{2}$ as the origin to that with $O_{1}$ as the origin). Hence, there is no need for us to resort to numerical integration even for a nonzero displacement $d$ from $O_{1}$ to $O_{2}$; this is of particular importance because previous experience with the LSBRM [8] has revealed that the computational errors accumulated during numerical integration may lead to a deterioration in the accuracy of the data predicted by the resulting software. Page-length restrictions do not permit us to provide a list of all these closedform expressions here, but we have reproduced such details in [9] for reference by any interested researchers.

It has been established in [7] and [8] that the minimization of the positive-definite $\Delta$ with respect to the mode coefficients $p_{m}$ (where $m=1,2,3, \cdots, M$ ) and $q_{n}$ (where $n=1,2,3, \cdots, N$ ) results in the following inhomogeneous matrix equation:

$$
\underline{U x}=-\underline{v}
$$

where $\underline{x}=\left(p_{1} p_{2}, \cdots, p_{M} q_{1} q_{2}, \cdots, q_{N}\right)^{t}$ is actually $y$ without the input $p_{0}$ coefficient, and where the $(M+N) \times(M+N) \underline{U}$ and $(M+N) \times 1 \underline{v}$ submatrices are obtained by partitioning $\underline{W}$ in the following manner:

$$
\underline{W}=\left[\begin{array}{cc}
w_{11} & \underline{v}^{*} \\
\underline{v} & \underline{U}
\end{array}\right]
$$

Since $\underline{U}$ is obviously a positive-definite Hermitian matrix as well, we may choose to employ the efficient Cholesky routine (without matrix inversion) to solve (3) for $\underline{x}$ so as to derive the values of all the unknown mode coefficients with reference to the input $p_{0}$, of which only $p_{1} / p_{0}$ (for each of the eigenmodes) is required for the computation of the scattering-matrix entries via the following conversion formulas:

$$
\begin{aligned}
& s_{11}=s_{22}=\frac{1}{2}\left\{\left(\frac{p_{1}}{p_{0}}\right)_{\text {even }}+\left(\frac{p_{1}}{p_{0}}\right)_{\text {odd }}\right\} \\
& s_{12}=s_{21}=\frac{1}{2}\left\{\left(\frac{p_{1}}{p_{0}}\right)_{\text {even }}-\left(\frac{p_{1}}{p_{0}}\right)_{\text {odd }}\right\} .
\end{aligned}
$$

\section{SAmple Results}

As has been pointed out in Section I, Shen and MacPhie [6] have recently used the CCPT to analyze the thick eccentric iris. It is evident from the graphs in Fig. 3 (of the scattering coefficients $s_{11}$ and $s_{21}$ against the iris thickness $t$ ) that the results predicted by our LSBRM model compare favorably with those obtained by the CCPT version-for magnitude as well as phase. One of the main assumptions made by Shen and MacPhie is that "no modes can propagate in the iris waveguide" [6, p. 2641]; i.e., their iris functions as a cutoff waveguide and the amount of power that is able to tunnel through it must progressively decrease as its thickness is increased. This explains the monotonic behavior of the graphs in Fig. 3, where

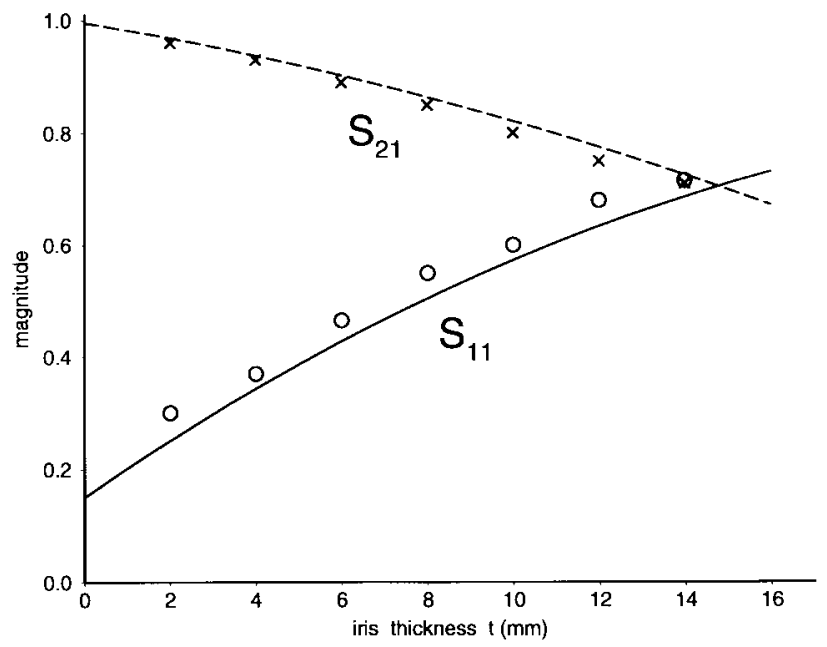

(a)

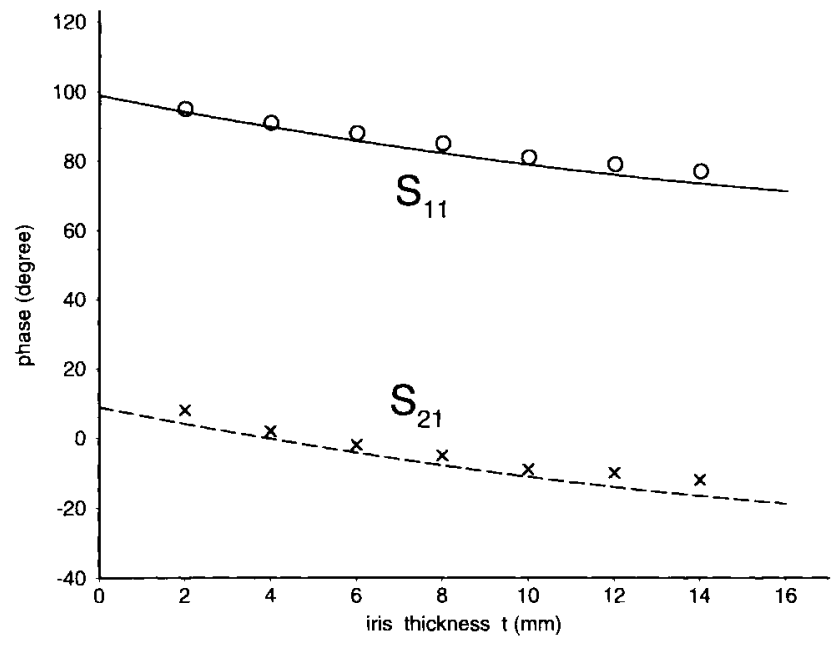

(b)

Fig. 3. Plots of: (a) magnitudes and (b) phases of scattering coefficients against iris thickness predicted by present LSBRM mode (- for $s_{11}$ and -..- for $s_{21}$ ) and CCPT model of Shen and MacPhie [6] (o o o for $s_{11}$ and $\times \times \times$ for $\left.s_{21}\right)$ for thick eccentric iris with $a=12.7 \mathrm{~mm}, b=9.5 \mathrm{~mm}$, $d=1.5 \mathrm{~mm}, \theta=0^{\circ}, f=9 \mathrm{GHz}$.

$\left|s_{11}\right|$ and $\left|s_{21}\right|$ will, in the limit, approach the expected values of unity and zero, respectively, with increasing $t$.

During the derivation of our model in Section II, we have not found it necessary to utilize the cutoff iris-waveguide assumption employed in [6]. Table I presents some of the results we painstakingly computed for the special case of a thick eccentric iris inserted into a circular waveguide (with $k_{o} a=3.5$ ) that supports the $\mathrm{TE}_{11}, \mathrm{TM}_{01}$ and $\mathrm{TE}_{21}$ modes. For this particular over-moded example, the $\mathrm{TE}_{11}$ and $\mathrm{TM}_{01}$ modes can propagate within the iris waveguide (with $k_{o} b=2.6$ ). It is, therefore, not surprising to note that we need to select large $M$ and $N$ settings for the two expansions in order to attain convergence in the results predicted by our LSBRM model for the amounts of scattered power carried by the reflected and transmitted modes in the

$$
\Delta=\left\{\begin{array}{l}
\iint_{S_{\text {apcr }}}\left|\sum_{m=1}^{m=M}\left\{p_{o} \delta_{m-1}+p_{m}\right\} \underline{e}_{m}^{\mathrm{I}}-\sum_{n=1}^{n=N} q_{n}\left\{1+(-1)^{K} \exp \left(-\gamma_{n} t\right)\right\} \underline{e}_{n}^{\Pi}\right|^{2} d S \\
\quad+\iint_{S_{\text {metal }}}\left|\sum_{m=1}^{m=M}\left\{p_{0} \delta_{m-1}+p_{m}\right\} \underline{e}_{m}^{\mathrm{I}}\right|^{2} d S \\
\quad+Z^{2} \iint_{S_{\text {apcr }}}\left|\sum_{m=1}^{m=M}\left\{p_{o} \delta_{m-1}-p_{m}\right\} \underline{h}_{m}^{\mathrm{I}}-\sum_{n=1}^{n=N} q_{n}\left\{1-(-1)^{K} \exp \left(-\gamma_{n} t\right)\right\} \underline{h}_{n}^{\Pi}\right|^{2} d S
\end{array}\right.
$$


TABLE I

Convergence of Numerical Results Obtained by Present LSBRM Model for Scattered Powers Carried by $\mathrm{TE}_{11}, \mathrm{TM}_{01}$ AND TE 21 Modes For Over-Moded THICK ECCENTRIC IRIS WITH $k_{o} a=3.5, k_{o} b=2.6, t / a=0.5$, $d / a=0.2, \theta=0^{\circ}$ (N.B.: For Convenience, Input Power From TE 11 Source has BeEn Normalized to Unity)

\begin{tabular}{|c|c|c|c|c|c|c|c|}
\hline \multirow{2}{*}{$\begin{array}{c}\text { Number of modes } \\
\qquad M=N\end{array}$} & \multicolumn{3}{|c|}{ Power carried by each reflected mode } & \multicolumn{3}{|c|}{ Power carried by each transmitted mode } & \multirow{2}{*}{$\begin{array}{l}\text { Sum of reflected and } \\
\text { transmitted powers }\end{array}$} \\
\hline & $\mathrm{TE}_{11}$ & $\mathrm{TM}_{01}$ & $\mathrm{TE}_{21}$ & $\mathrm{TE}_{11}$ & $\mathrm{TM}_{01}$ & $\mathrm{TE}_{21}$ & \\
\hline 10 & 0.046 & 0.107 & 0.026 & 0.468 & 0.036 & 0.028 & 0.711 \\
\hline 50 & 0.036 & 0.115 & 0.025 & 0.589 & 0.044 & 0.030 & 0.839 \\
\hline 100 & 0.034 & 0.117 & 0.025 & 0.633 & 0.048 & 0.032 & 0.889 \\
\hline 500 & 0.030 & 0.120 & 0.024 & 0.707 & 0.051 & 0.035 & 0.967 \\
\hline 1,000 & 0.029 & 0.120 & 0.024 & 0.727 & 0.052 & 0.037 & 0.989 \\
\hline 1,500 & 0.029 & 0.120 & 0.024 & 0.728 & 0.052 & 0.037 & 0.990 \\
\hline
\end{tabular}

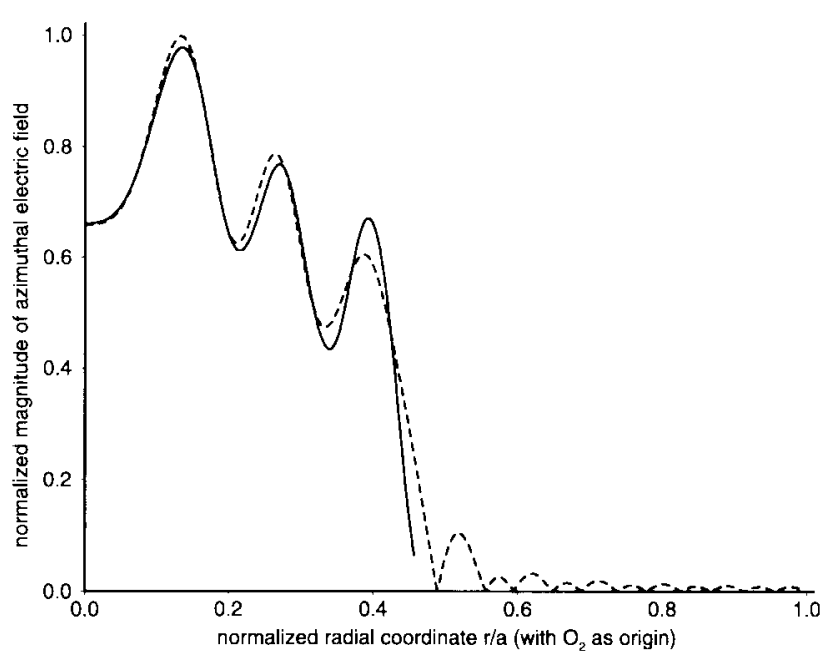

(a)

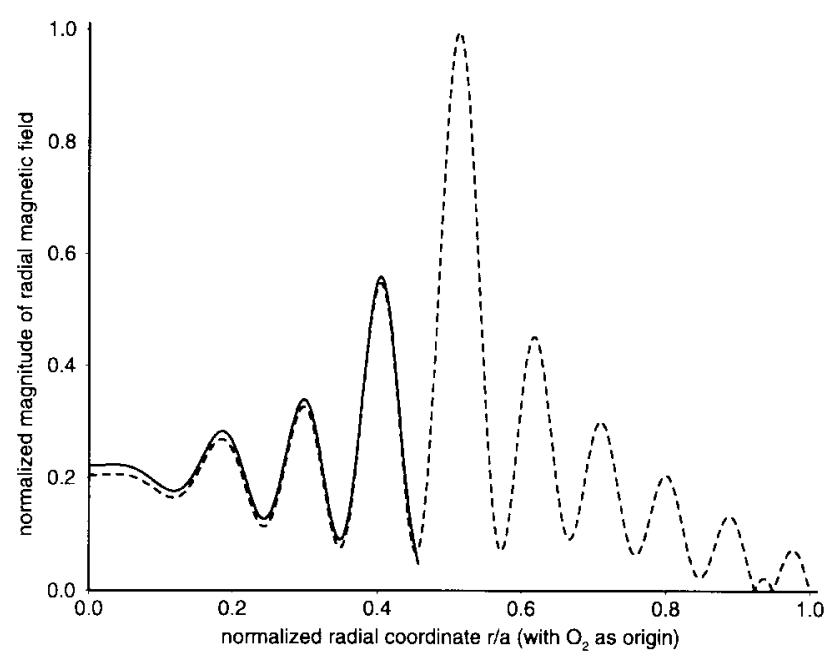

(b)

Fig. 4. Plots of transverse: (a) electric- and (b) magnetic-field components against normalized radial coordinate over discontinuity plane at $z=-1 / 2 t$ (- for Region I and - - - for Region II) for thick eccentric iris with $k_{o} a=2.5$, $b / a=t / a=0.5, d / a=0.2, \theta=0^{\circ}$.

$z<-1 / 2 t$ and $z>1 / 2 t$ sections, respectively. The power of the $\mathrm{TE}_{11}$ wave incident on this loss-free structure has, for convenience, been normalized to unity, and we observe from the last column of
Table I that the sum of the scattered powers converges to within $1 \%$ of this input-power value if we increase $M$ and $N$ to more than 1500 for this over-moded situation.

Another important advantage of our LSBRM model is that we are also able to generate the electromagnetic-field plots in the vicinity of the iris. This is possible because the solution of (3) additionally yields the values of all the other mode coefficients for the expansions we adopted in (1) to represent the fields in Regions I and II. Fig. 4 provides a typical set of electric- and magnetic-field plots at $z=-1 / 2 t$ for one such eccentric iris. There is excellent match over $S_{\text {aper }}$ between the fields of the two expansions, and the electric fields tangential to $S_{\text {metal }}$ have negligibly small magnitudes. In general, currents will flow on the conducting surfaces and the transverse components of the magnetic field in Fig. 4(b) are, thus, not small in magnitude over $S_{\text {metal }}$.

\section{CONCLUSIONS}

We have successfully demonstrated that the mathematically rigorous LSBRM can be employed to develop a reliable computer model that is able to accurately predict the scattering characteristics of the thick circular iris sited nonconcentrically within the circular waveguide. In contrast to that reported by Shen and MacPhie [6], our model does not utilize any cutoff iris-waveguide assumption, and there is consequently no rigid validity limit on the operating frequency range; however, there is the need to select larger $M$ and $N$ settings if we want to ensure the onset of convergence in the numerical results at the higher operating frequencies. Another useful feature is that field plots are readily generated without too much additional effort (once (3) has been solved via the computationally efficient Cholesky routine for the decomposition of positive-definite Hermitian matrices).

Hence, we may confidently set about using our resulting model (which is suitable for running on a workstation or its equivalent) to analyze and design thick eccentric irises for "applications in matched windows in circular waveguide [5], in constructing filters as a block element, and in the coupling to a dual-mode circular cavity by a circular hole," as has been envisaged in [6, p. 2641].

\section{REFERENCES}

[1] G. L. Ragan, Microwave Transmission Circuits. New York: McGrawHill, 1948.

[2] N. Marcuvitz, Waveguide Handbook. New York: McGraw-Hill, 1951.

[3] G. L. James, "Admittance of irises in coaxial and circular waveguides for $\mathrm{TE}_{11}$-mode excitation," IEEE Trans. Microwave Theory Tech., vol. MTT-35, pp. 430-434, Apr. 1987. 
[4] R. W. Scharstein and A. T. Adams, "Thick circular iris in a TE 11 -mode circular waveguide," IEEE Trans. Microwave Theory Tech., vol. 36, pp. 1529-1531, Nov. 1988.

[5] L. Carin, K. J. Webb, and S. Weinreb, "Matched windows in circular waveguide," IEEE Trans. Microwave Theory Tech., vol. 36, pp. 1359-1362, Sept. 1988.

[6] Z. Shen and R. H. MacPhie, "Scattering by a thick off-centered circular iris in circular waveguide," IEEE Trans. Microwave Theory Tech., vol. 43, pp. 2639-2642, Nov. 1995.

[7] J. B. Davies, "A least-squares boundary residual method for the numerical solution of scattering problems," IEEE Trans. Microwave Theory Tech., vol. MTT-21, pp. 99-104, Feb. 1973.

[8] A. L. Cullen and S. P. Yeo, "Using the least-squares boundary residual method to model the symmetrical five-port waveguide junction," Proc. Inst. Elect. Eng., vol. 134, pt. H, Apr. 1987, pp. 116-124.

[9] S. G. Teo, "Analysis of circular-waveguide iris," M. Eng. thesis, Dept. Elect. Eng., National Univ. Singapore, Kent Ridge, Singapore, 1997.

\section{Development of an Improved Two-Dimensional Finite-Element Code for Cylindrically Symmetric Eigenmodes}

\author{
Kai Masuda, Kiyoshi Yoshikawa, Masami Ohnishi, \\ Yasushi Yamamoto, Hisayuki Toku, \\ Masaaki Sobajima, and Jiro Kitagaki
}

\begin{abstract}
A new two-dimensional finite-element (FE) eigenmode solver has been developed, which is suitable for calculating cylindrically symmetric modes. The quantity $H_{\theta} / r$ is used in the code to describe the electromagnetic fields instead of $H_{\theta}$ or $r H_{\theta}$, which is preferentially used in the existing codes, and the new formulation with $H_{\theta} / r$ is found to show higher accuracy and smoother convergence with respect to the number of mesh points. Comparison is also made between linear and quadratic elements, resulting in remarkably higher accuracy by the latter.
\end{abstract}

Index Terms-Cavity eigenmode, finite-element method.

\section{INTRODUCTION}

Cylindrically symmetric cavities are utilized in many radiofrequency (RF) devices, such as klystrons, RF guns, and various accelerating structures in particle accelerators. Many computer codes [1]-[11] have been developed thus far, and are in use for RF cavity designing for more than 30 years.

For cylindrically symmetric standing-wave modes, probably the most commonly used code would be the SUPERFISH [2], which calculates eigenfrequencies and corresponding angular magnetic field $H_{\theta}$ at the mesh points using the finite-differential method (FDM) with triangular meshes. However, depending on the cavity geometry, it is sometimes not accurate enough or, in other words, takes too much central processing unit (CPU) time and computer memory to achieve required accuracy. Since both higher accuracy and less computational efforts are always important from the viewpoint of saving time and effort for the users, continuous improvements of greater extent are called for in specific problems.

Manuscript received May 19, 1997; revised March 16, 1997.

The authors are with the Institute of Advanced Energy, Kyoto University, Gokasho, Uji, Kyoto 611, Japan.

Publisher Item Identifier S 0018-9480(98)05498-2.
From this viewpoint, a new two-dimensional-code Kyoto University eigenmode solver (KUEMS) has been developed, which is aimed at improved calculations of cylindrically symmetric $\mathrm{TM}_{0} \mathrm{~nm}$ modes applicable to klystron simulations [12]. Instead of $H_{\theta}$ or $r H_{\theta}$ preferentially used in the existing codes [1]-[3], [7], the KUEMS uses: the quantity $H_{\theta} / r$ to describe the electromagnetic fields, which has the advantage of not requiring any boundary conditions on the symmetry axis, and the finite-element method (FEM) with quadratic triangular elements, which has high capability to model arbitrary structures.

This paper describes the numerical methods used in the KUEMS, followed by comparisons of the numerical results among the three different formulations, i.e., with $H_{\theta} / r, H_{\theta}$, and $r H_{\theta}$, to show the advantageous features of this new formulation with $H_{\theta} / r$ with respect to accuracy in the eigenfrequencies, and convergence of the electric field on the symmetry axis. Comparisons between the linear and quadratic elements are also made to examine the accuracy together with the SUPERFISH.

\section{Numerical Methods IN THE KUEMS}

The numerical methods used in the KUEMS are described in this section, including the new FE formulation with the quantity $H_{\theta} / r$. The essential difference from the other formulations with $H_{\theta}$ and $r H_{\theta}$ is described in Section II-B.

\section{A. Basic Equations and Weak Formulation}

For resonant electromagnetic fields, we can assume electric and magnetic fields $\boldsymbol{E}$ and $\boldsymbol{H}$ at a time $t$ and location $\boldsymbol{r}$ by

$$
\begin{aligned}
\boldsymbol{E}(\boldsymbol{r}, t) & =e(\boldsymbol{r}) \operatorname{Re}[\exp (i \omega t)], \\
\boldsymbol{H}(\boldsymbol{r}, t) & =\sqrt{\epsilon_{0} / \mu_{0}} \boldsymbol{h}(\boldsymbol{r} \operatorname{Re}[i \exp (i \omega t)]
\end{aligned}
$$

where $\epsilon_{0}$ and $\mu_{0}$ are the permittivity and permeability in free space, $f=\omega / 2 \pi$ is the resonant frequency, and $\boldsymbol{e}$ and $\boldsymbol{h}$ are the eigenmode patterns. Then, from Maxwell's equations in free space, the frequency and the magnetic field are expressed by the following eigenvalue problem:

$$
\begin{aligned}
\nabla \times \nabla \times \boldsymbol{h} & =k^{2} \boldsymbol{h} \text { in } \Omega \\
\boldsymbol{n} \times(\nabla \times \boldsymbol{h}) & =\mathbf{0} \text { on } \Gamma
\end{aligned}
$$

and the corresponding electric field is given by

$$
\boldsymbol{e}=\frac{1}{k} \nabla \times \boldsymbol{h} \text { in } \Omega
$$

where $k=\omega / c$, the boundary $\Gamma$, and the domain $\Omega$ are the inner surface of the cavity wall and its volume, respectively, and $\boldsymbol{n}$ is the unit vector normal to $\Gamma$.

Satisfaction of both (2) and (3) is equivalent to the following formulation:

$$
\begin{array}{r}
\int_{\Omega} \boldsymbol{v} \cdot\left[k^{2} \boldsymbol{h}-\nabla \times(\nabla \times \boldsymbol{h})\right] d v+\int_{\Gamma} \boldsymbol{v} \cdot[\boldsymbol{n} \times(\nabla \times \boldsymbol{h})] d S=0 \\
\text { for any } \boldsymbol{v}
\end{array}
$$

where $d V$ and $d S$ are a volume element in $\Omega$ and a surface element on $\Gamma$, respectively. With Gauss' divergence theorem, (5) can be reduced to the following well-known weak formulation [8]:

$$
\int_{\Omega}(\nabla \times \boldsymbol{v}) \cdot(\nabla \times \boldsymbol{h}) d V=k^{2} \int_{\Omega} \boldsymbol{v} \cdot \boldsymbol{h} d V, \quad \text { for any } \boldsymbol{v} .
$$

